\title{
Antimicrobial Susceptibility Characteristics and Risk Factors Associated with Adult Sepsis in Wenzhou, China
}

\author{
Xiaoxiao Qu $\mathbb{D}^{1, *}$, Shishi Wang ${ }^{\prime} *$, Ying Qu $\mathbb{D}^{2}$, Huiyan Wang', Xuanmei Ye', Luming Tang ${ }^{3}$, Qipeng Xie $\mathbb{D}^{\prime}$ \\ 'Department of Clinical Laboratory, The Second Affiliated Hospital \& Yuying Children's Hospital of Wenzhou Medical University, Wenzhou, Zhejiang, \\ 325027, People's Republic of China; ${ }^{2}$ Department of Clinical Laboratory, Wenzhou People's Hospital, The Third Affiliated Hospital of Shanghai \\ University, The Third Clinical Institute Affiliated to Wenzhou Medical University, Wenzhou, Zhejiang, 325035, People's Republic of China; ${ }^{3}$ Emergency \\ Department, The Second Affiliated Hospital \& Yuying Children's Hospital of Wenzhou Medical University, Wenzhou, Zhejiang, 325027, People's \\ Republic of China \\ *These authors contributed equally to this work
}

Correspondence: Qipeng Xie, Department of Clinical Laboratory, The Second Affiliated Hospital \& Yuying Children's Hospital of Wenzhou Medical University, Wenzhou, Zhejiang, 325035, People’s Republic of China, Tel +86 I5I 577 87I59, Email pandon2002@I63.com

Purpose: To clarify the distribution of pathogenic bacteria by analyzing the bacterial susceptibility characteristics and risk factors for adult sepsis in The Wenzhou city, Zhejiang province, China, and to aid early diagnosis, monitoring, and prognosis prediction in cases of bacterial sepsis.

Patients and Methods: We retrospectively analyzed 329 patients with sepsis admitted to the Second Affiliated Hospital of Wenzhou Medical University between January 2018 and March 2021. Laboratory data were collected before and after treatment; moreover, the bacterial susceptibility characteristics and risk factors for sepsis were comprehensively analyzed using the Sequential Organ Failure Assessment (SOFA) score.

Results: The SOFA score was negatively correlated with the prognosis $(\mathrm{P}<0.05)$. We isolated 47 pathogenic strains from blood culture samples, including 29 gram-positive strains, 18 gram-negative strains. The most common gram-negative pathogens in blood cultures are Klebsiella pneumoniae and Escherichia coli, while the most common gram-positive pathogens are Staphylococcus aureus and Staphylococcus hominis. Gram-negative pathogens had resistance rates of $77 \%$ and $62.5 \%$ to ciprofloxacin and ceftriaxone, respectively. Gram-positive bacteria had a high resistance to penicillin at $100 \%$. Prognostic factors for sepsis included patients' consciousness, SOFA score, prothrombin time, international normalized ratio, fibrinogen, D-dimer, and aspartate aminotransferase $(\mathrm{P}<0.05)$. Of these, the D-dimer level could predict the outcome of patients with sepsis $(\mathrm{AUC}=0.661, \mathrm{P}<0.05)$.

Conclusion: The pathogens detected in adult sepsis in Wenzhou are mainly Klebsiella pneumoniae, Escherichia coli, Staphylococcus aureus, and Staphylococcus hominis. The pathogens exhibited differences in drug susceptibility. The optimal antibiotics should be chosen based on the principles of rational use and drug susceptibility. Combined with D-dimer levels, these parameters can be used to determine the optimal strategy for preventing and treating pathogenic bacteria.

Keywords: adult sepsis, drug susceptibility characteristics, risk factors, D-dimer

\section{Introduction}

According to the international consensus definition of sepsis and septic shock (Sepsis 3.0), sepsis is a life-threatening organ dysfunction caused by a dysregulated host response to infection. ${ }^{1}$ Sepsis is characterized by inflammatory response and coagulation dysfunction, which causes inadequate perfusion of organs and tissues, leading to multiple organ dysfunction syndrome and even death. ${ }^{2}$ Sepsis is a serious global health problem and the leading cause of morbidity and mortality in intensive care units (ICUs) worldwide, ${ }^{3}$ with approximately 49 million new cases and 11 million deaths worldwide in $2017 .{ }^{4}$ 
Prompt diagnosis of sepsis is critical since delayed appropriate treatment can severely affect the outcomes. Currently, diagnosis is established based on clinical characteristics and blood tests for detecting inflammation-related blood biomarkers, including C-reactive protein (CRP) and procalcitonin (PCT). However, these clinically available protein biomarkers lack specificity, ${ }^{5}$ which impedes the early identification of sepsis. Microbial culture technology remains the gold standard for identifying the phenotype of pathogenic pathogens. However, it takes up to $72 \mathrm{~h}$ and has a high false-negative rate. ${ }^{6}$

The aim of this retrospective study was to examine the clinical characteristics, bacterial susceptibility and risk factors of patients with sepsis in Wenzhou area. In addition, we aimed to provide data for early clinical diagnosis as well as timely and accurate antibiotic selection in disease surveillance.

\section{Objects and Methods}

\section{Patients}

This retrospective study included 329 adult patients ( $>18$ years old) with sepsis admitted to the Second Affiliated Hospital of Wenzhou Medical University between January 2018 and March 2021, and this hospital has a bed capacity of 2667. This study was approved by the Ethics Committee of the Second Affiliated Hospital of Wenzhou Medical University (Approval No: LCKY2020-421). Patients with sepsis were classified into mild, moderate and severe according to organ dysfunction scores (Acute Physiological and Chronic Health Evaluation [APACHE II] and Sequential Organ Failure Assessment [SOFA]). Laboratory parameters (eg, PCT, CRP, cytokines, IL-8, etc.) were collected from septic patients, as well as microbiological characteristics and drug resistance in blood cultures.

\section{Inclusion and Exclusion Criteria}

The inclusion criteria were as follows: a) patients with sepsis meeting the diagnostic criteria in the Guidelines for Emergency Treatment of Sepsis/Septic Shock in China (2018), ${ }^{7}$ ie, having a Sequential Organ Failure Assessment (SOFA) score $\geq 2 ;{ }^{1,8}$ b) having complete clinical information and test data, available for statistical analysis; and c) being fully aware of the research protocol and voluntarily accepting the index inspection. The exclusion criteria were as follows: a) inability to take antimicrobial drugs within 1 month before enrollment; b) having coagulation dysfunction or tendency; c) being complicated with active bleeding; d) pregnancy; and e) having mental illness or confusion and being unable to cooperate with the test.

\section{Prognostic Criteria}

Based on the international guidelines for managing sepsis and septic shock, ${ }^{9}$ invalid (aggravation) is defined as persistence or worsening of the selected symptoms and signs during or after treatment, or appearance of new symptoms or signs and/or use of the other antimicrobial drugs for the disease. Cure (improvement) was defined as disappearance/complete recovery of symptoms and signs, as well as normal imaging and laboratory examination results, at the end of treatment.

\section{Instruments and Methods}

For bacterial culture and identification, we used the automatic microbial culture system (Bact/ALERT 3D) as well as its supporting GP identification and ASTGN13 drug-sensitive card; Colombian blood AGAR culture solution. Routine blood tests including HB, PLT, CRP, WBC, NEU, and LYM were performed using Mindray BC5310 and BC5390 AUTOMATIC blood cell analyzer CRP integrated machine. The related reagents, quality control products, and calibration products were provided by Shenzhen Mindray Electronics Co., Ltd. Biochemical analysis including PA, ALT, AST, and CREA were performed using a Siemens automatic biochemical apparatus; further, immunochemistry analysis was performed using a Roche COBAS P612 apparatus while coagulation (PT, APTT, INR, FIB, D-D) was measured using the STAGO apparatus. For bacterial culture and identification, we used the automatic microbial culture system (Bact/ALERT 3D) as well as its supporting GP identification and ASTGN13 drug-sensitive card; Colombian blood AGAR culture solution.

\section{Strain Identification and Drug Sensitivity Test}

Blood culture specimens are inoculated on Columbia blood plates and incubated for 24-72 h. If positive, the pathogenic bacteria are isolated and identified; if negative, no bacterial growth is reported. Samples were isolated and cultured 
following the National Clinical Examination Procedures; additionally, the bacteria were identified using a MALDI Biotyper (Bruker) automatic rapid microbial mass spectrometer. Regarding the automatic drug sensitivity system test, the minimum inhibitory concentration was detected based on the VITEK 2 Compact system and Walk-Away specimen processor. Regarding the K-b disk diffusion method, Vernier calipers were used to measure the diameter of the bacteriostatic ring, with the results being compared with the interpretation standard. The standards referred to the National Clinical Testing Procedures ${ }^{10}$ and CLSI Performance standards for antimicrobial susceptibility testing. ${ }^{11}$

\section{Statistical Analysis}

Plotting was performed using GraphPad Prism 7 software. Statistical analyses were performed using SPSS26.0 software. Mean \pm standard deviation was used to represent the normal distribution of clinical data. Between-group comparisons were performed using a $t$-test. Statistical data were expressed as the percentage or number of cases using the $\chi^{2}$ test or Fisher's exact probability method. Receiver operating characteristic (ROC) curve analysis was used to analyze the predictive effect of D-dimer on sepsis outcome. Statistical significance was set at $\mathrm{P}<0.05$.

\section{Results}

\section{Basic Information of the Included Participants}

A retrospective analysis was performed on 329 patients with sepsis admitted to the Second Affiliated Hospital of Wenzhou Medical University from January 2018 to March 2021. There were 174 (52.9\%) male patients with sepsis; among them, improvement was noted in $140(54.5 \%)$ whereas the condition aggravated or resulted in death for 34 patients $(47.2 \%)$. Further, 155 (47.1\%) female patients had sepsis; the condition improved in $117(45.5 \%)$ of these, whereas the condition aggravated or resulted in the death for 38 patients $(52.8 \%)$. There were 142 cases (43.3\%) with hypertension, 186 cases $(56.7 \%)$ without hypertension, 78 cases $(23.8 \%)$ with diabetes, and 250 cases $(76.2 \%)$ without diabetes. There were no significant between-group differences in sex, age, smoking, alcohol drinking, or other clinical data. As shown in Table 1, the outcome of sepsis was associated with consciousness $(\mathrm{P}<0.05)$.

\section{Association of Clinical Features with Patient Prognosis}

According to the outcome of sepsis, patients with sepsis were divided into improvement, aggravation, and death groups.

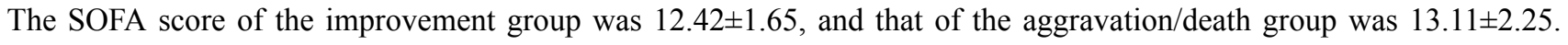
The higher the score, the worse the prognosis $(\mathrm{P}<0.05)$, while other indicators such as body temperature, systolic blood pressure, diastolic blood pressure and mean arterial pressure did not show significant differences, as shown in Table 2.

Table I General Information on Patients with Sepsis

\begin{tabular}{|c|c|c|c|c|c|c|}
\hline \multirow[t]{2}{*}{ Indicators } & \multirow[t]{2}{*}{ Grouping } & \multirow[t]{2}{*}{ Number of Cases (\%) } & \multicolumn{2}{|c|}{ Outcomes } & \multirow[t]{2}{*}{$\chi^{2} / \mathrm{t}$} & \multirow[t]{2}{*}{$\mathbf{P}$} \\
\hline & & & Amelioration $(n=257)$ & Aggravation/Death $(n=72)$ & & \\
\hline \multirow[t]{2}{*}{ Gender } & Male & $174(52.9)$ & $140(54.5)$ & $34(47.2)$ & 1.187 & 0.276 \\
\hline & Female & $155(47.1)$ & II (45.5) & $38(52.8)$ & & \\
\hline Age & & & $58.98 \pm 17.67$ & $61.18 \pm 16.44$ & -0.948 & 0.344 \\
\hline \multirow[t]{2}{*}{ Smoking } & No & $298(90.9)$ & $234(91.1)$ & $64(90.1)$ & 0.055 & 0.814 \\
\hline & Yes & $30(9.1)$ & $23(8.9)$ & $7(9.9)$ & & \\
\hline \multirow[t]{2}{*}{ Alcohol drinking } & No & $299(91.2)$ & $234(91.1)$ & $65(91.5)$ & 0.017 & 0.896 \\
\hline & Yes & $29(8.8)$ & $23(8.9)$ & $6(8.5)$ & & \\
\hline \multirow[t]{2}{*}{ Hypertension } & No & $186(56.7)$ & $146(56.8)$ & $40(56.3)$ & 0.005 & 0.943 \\
\hline & Yes & $142(43.3)$ & III (43.2) & 31 (43.7) & & \\
\hline \multirow[t]{2}{*}{ Diabetes } & No & $250(76.2)$ & $196(76.3)$ & $54(76.1)$ & 0.001 & 0.971 \\
\hline & Yes & $78(23.8)$ & $61(23.7)$ & $17(23.9)$ & & \\
\hline \multirow[t]{2}{*}{ Consciousness } & Clear & $317(96.4)$ & $25 \mathrm{I}(97.7)$ & 66 (91.7) & 5.759 & 0.016 \\
\hline & Not clear /vague & $12(3.6)$ & $6(2.3)$ & $6(8.3)$ & & \\
\hline
\end{tabular}


Table 2 Association of Clinical Features with Patient Prognosis

\begin{tabular}{|l|l|l|l|l|}
\hline \multirow{2}{*}{ Indicators } & \multicolumn{2}{|c|}{ Outcomes } & \multirow{2}{*}{ t/z } \\
\cline { 2 - 4 } & Amelioration (n=257) & Aggravation/Death (n=72) & \\
\hline Body temperature & $37.35(36.90,38.20)$ & $37.50(36.80,38.10)$ & -0.346 & 0.729 \\
Systolic blood pressure & $123.32 \pm 21.78$ & $119.94 \pm 22.22$ & 1.157 & 0.248 \\
Diastolic blood pressure & $73.15 \pm 13.18$ & $71.71 \pm 11.83$ & 0.837 & 0.403 \\
Mean arterial pressure & $89.57 \pm 15.23$ & $87.05 \pm 15.05$ & 1.246 & 0.214 \\
SOFA score & $12.42 \pm 1.65$ & $13.11 \pm 2.25$ & -2.429 & 0.017 \\
\hline
\end{tabular}

\section{Association of Laboratory Indicators with Patient Prognosis}

Table 2 shows the laboratory indicators of all the patients. As shown in Table 3, sepsis was correlated with PT, INR, FIB, D-dimer, and AST $(\mathrm{P}<0.05)$ but not with hemoglobin $(\mathrm{HB})$, platelet count $(\mathrm{PLT})$, activated partial thrombin time (APTT), CRP, PCT, prealbumin, ALT, and creatinine (all P > 0.05).

\section{Comparison of Blood Culture Results}

The positive rate of blood culture was $46 \%$, with gram-negative and gram-positive bacteria having positive rates of $18 \%$ and $28 \%$, respectively. There were no significant differences between amelioration and aggravation groups $(\mathrm{P}<0.05)$. See Table 4.

\section{Pathogen Distribution}

A total of 47 pathogenic strains were isolated from blood culture samples, including 29 (61.7\%) and 18 (38.3\%) gram-positive and gram-negative. The most common gram-negative pathogens in blood culture were Klebsiella pneumoniae and Escherichia coli, while the most common gram-positive pathogens were Staphylococcus aureus and Staphylococcus hominis (Table 5).

\section{Drug Susceptibility Analysis of Gram-Positive Cocci}

Drug susceptibility analysis showed a high level of resistance to penicillin (up to 100\%) in Gram-positive cocci and a low level of resistance to rifampicin, vancomycin and linezolid. The specific results are shown in Table 6.

Table 3 Association of Laboratory Indicators with Patient Prognosis

\begin{tabular}{|c|c|c|c|c|}
\hline \multirow[t]{2}{*}{ Indicators/Reference Range } & \multicolumn{2}{|c|}{ Outcomes } & \multirow[t]{2}{*}{$t / \mathbf{z}$} & \multirow[t]{2}{*}{$\mathbf{P}$} \\
\hline & Amelioration $(n=257)$ & Aggravation/Death $(n=72)$ & & \\
\hline HB $(135 \sim 175 \mathrm{~g} / \mathrm{L})$ & $123.67 \pm 23.80$ & $118.19 \pm 22.62$ & 1.742 & 0.082 \\
\hline PLT $\left(125 \sim 350 \times 10^{9} / \mathrm{L}\right)$ & $169.00(133.00,239.75)$ & $155.00(92.25,238.00)$ & -1.944 & 0.052 \\
\hline PT (I2 15 seconds) & $14.70(13.70,15.90)$ & $15.00(14.10,18.00)$ & -2.919 & 0.004 \\
\hline APTT (30 45 seconds) & $42.80(36.70,49.08)$ & $44.00(38.20,51.08)$ & -1.544 & 0.123 \\
\hline INR (0.85-I.I5) & $1.17(1.08,1.29)$ & $1.20(1.13,1.50)$ & -2.962 & 0.003 \\
\hline FIB $(2 \sim 4 g / L)$ & $5.63(4.23,6.96)$ & $4.83(3.71,6.65)$ & -2.203 & 0.028 \\
\hline $\mathrm{D}-\mathrm{D}(0 \sim 0.5 \mu \mathrm{g} / \mathrm{mL})$ & $\mathrm{I} .53(0.78,3.3 \mathrm{I})$ & $3.46(1.27,8.66)$ & -4.129 & $<0.001$ \\
\hline CRP (In hospital) (0 8mg/L) & $|17.5|(62.50,190.07)$ & $107.27(52.90,210.00)$ & -0.010 & 0.992 \\
\hline WBC (3.5 9.5 $\left.\times 10^{\wedge} 9 / \mathrm{L}\right)$ & $10.40(6.7 \mathrm{I}, \mathrm{I5.02})$ & $10.73(6.60,14.91)$ & -0.472 & 0.637 \\
\hline NEU (0.4 0.75) & $0.84(0.75,0.90)$ & $0.83(0.76,0.88)$ & -0.895 & 0.371 \\
\hline $\operatorname{LYM}(0.2 \sim 0.5)$ & $0.10(0.05,0.16)$ & $0.10(0.05,0.16)$ & -0.646 & 0.518 \\
\hline PCT $(0 \sim 0.046 \mathrm{ng} / \mathrm{mL})$ & $1.50(0.38,7.50)$ & $5.89(0.32,27.31)$ & -1.689 & 0.091 \\
\hline PA (250 400mg/L) & $|34.17 \pm 62.2|$ & $118.78 \pm 63.88$ & 1.706 & 0.089 \\
\hline ALT (9 50U/L) & $24.00(14.00,50.00)$ & $34.50(16.00,73.75)$ & -1.730 & 0.084 \\
\hline AST (I5 40U/L) & $34.00(19.00,72.00)$ & $47.00(26.75,130.25)$ & -3.063 & 0.002 \\
\hline Crea $(57 \sim 97 \mu \mathrm{mol} / \mathrm{L})$ & $69.15(53.28,88.78)$ & $67.45(54.43,118.10)$ & -0.679 & 0.497 \\
\hline
\end{tabular}


Table 4 Comparison of Blood Culture Results of Patients with Sepsis

\begin{tabular}{|c|c|c|c|c|c|c|}
\hline \multirow[t]{2}{*}{ Indicators } & \multirow[t]{2}{*}{ Grouping } & \multirow[t]{2}{*}{ Number of Cases (\%) } & \multicolumn{2}{|c|}{ Outcomes } & \multirow[t]{2}{*}{$\chi^{2} / \mathrm{t}$} & \multirow[t]{2}{*}{$\mathbf{P}$} \\
\hline & & & Amelioration $(n=257)$ & Aggravation/Death $(n=72)$ & & \\
\hline \multirow[t]{2}{*}{ Blood culture } & Negative & $283(86.0)$ & $223(86.8)$ & $60(83.3)$ & 0.552 & 0.457 \\
\hline & Positive & $46(14.0)$ & $34(13.2)$ & $12(16.7)$ & & \\
\hline \multirow[t]{2}{*}{ G - bacteria } & No & $311(94.5)$ & $246(95.7)$ & $65(90.3)$ & 3.221 & 0.073 \\
\hline & Yes & $18(5.5)$ & II (4.3) & $7(9.7)$ & & \\
\hline \multirow[t]{2}{*}{ G + bacteria } & No & $30 I(91.5)$ & $234(91.1)$ & $67(93.1)$ & 0.290 & 0.590 \\
\hline & Yes & $28(8.5)$ & $23(8.9)$ & $5(6.9)$ & & \\
\hline
\end{tabular}

\section{Drug Susceptibility Analysis of Gram-Negative Bacteria}

Drug sensitivity analysis showed that Gram-negative bacteria were $100 \%$ susceptible to amikacin and $77 \%$ and $62.5 \%$ resistant to ciprofloxacin and ceftriaxone respectively. The specific results are shown in Table 7.

\section{Univariate and Multivariate Analysis of Risk Factors for Sepsis}

Univariate analysis showed that factors associated with sepsis outcome included the state of consciousness, SOFA score, PT, INR, FIB, D-dimer, and AST ( $\mathrm{P}<0.05)$, as shown in Tables $1-3$. Variables with $\mathrm{P}<0.2$ in univariate analysis and variables with clinical significance according to professionals were included in the logistic regression model. We found that D-dimer $[\mathrm{OR}=1.096,95 \% \mathrm{CI}(1.030-1.166), \mathrm{P}=0.004]$ was an independent predictor of sepsis outcome; however, state of consciousness, SOFA score, PT, INR, FIB, and AST were not predictors $(\mathrm{P}>0.05)$ (Table 8).

\section{Correlation Analysis of the Predictive Value of D-Dimers}

To further analyze the predictive utility of D-dimer on sepsis outcome, an ROC curve was plotted. We found that the specific D-2 level could predict sepsis outcome $(\mathrm{AUC}=0.661$, Figure 1). When the critical D-dimer level was $3.36 \mu \mathrm{g} /$

Table 5 Isolation Number and Composition Ratio of Pathogens in Blood Culture

\begin{tabular}{|l|l|l|}
\hline Pathogen & Frequency (n) & Percentages (\%) \\
\hline G - bacteria & $\mathbf{1 8}$ & $\mathbf{3 8 . 3}$ \\
Klebsiella pneumoniae & 8 & 17.1 \\
E. coli & 5 & 10.7 \\
Acinetobacter baumannii & $\mathrm{I}$ & 2.1 \\
Proteus mirabilis & $\mathrm{I}$ & 2.1 \\
Vibrio vulnificus & $\mathrm{I}$ & 2.1 \\
Anaerobic gram-negative bacteria & $\mathrm{I}$ & 2.1 \\
Gram-negative cocci & $\mathrm{I}$ & 2.1 \\
G + bacteria & $\mathbf{2 9}$ & $\mathbf{6 1 . 7}$ \\
Staphylococcus aureus & 6 & 12.8 \\
Staphylococcus hominis & 6 & 12.8 \\
Staphylococcus epidermidis & 5 & 10.6 \\
Staphylococcus haemolyticus & 2 & 4.3 \\
Staphylococcus capitis & 2 & 4.3 \\
Staphylococcus kongi & 2 & 4.3 \\
Pseudomonas aeruginosa & $\mathrm{I}$ & 2.1 \\
Listeria monocytogenes & $\mathrm{I}$ & 2.1 \\
Staphylococcus coli & $\mathrm{I}$ & 2.1 \\
Urealyticum & $\mathrm{I}$ & 2.1 \\
Gram-positive streptococcus & $\mathrm{I}$ & 2.1 \\
Anaerobic gram-positive cocci & $\mathrm{I}$ & 2.1 \\
\hline
\end{tabular}


Table 6 Susceptibility Rates of Clinically Isolated Gram-Positive Cocci to Antibiotics (\%)

\begin{tabular}{|l|l|l|l|}
\hline \multirow{2}{*}{ Antimicrobial Drugs } & \multicolumn{3}{|c|}{ Gram-Positive (n=22) } \\
\cline { 2 - 4 } & S & I & R \\
\hline Clindamycin & 36.4 & 0.0 & 63.6 \\
Erythromycin & 18.2 & 0.0 & 81.8 \\
Penicillin & 0.0 & 0.0 & 100.0 \\
Rifampicin & 100.0 & 0.0 & 0.0 \\
Tetracycline & 81.8 & 4.6 & 13.6 \\
Gentamicin & 86.3 & 4.6 & 9.1 \\
Ciprofloxacin & 31.8 & 0.0 & 68.2 \\
Levofloxacin & 31.8 & 0.0 & 68.2 \\
Cotrimoxazole & 50.0 & 0.0 & 50.0 \\
Vancomycin & 100.0 & 0.0 & 0.0 \\
Oxacillin & 63.6 & 0.0 & 36.4 \\
Linezolid & 100.0 & 0.0 & 0.0 \\
\hline
\end{tabular}

Table 7 Susceptibility Rate of Main Isolated Gram-Negative Bacteria to Antibiotics (\%)

\begin{tabular}{|l|l|l|l|}
\hline \multirow{2}{*}{ Antimicrobial Drugs } & \multicolumn{3}{|c|}{ Gram-Negative (n= 13) } \\
\cline { 2 - 4 } & S & I & R \\
\hline Amikacin & 100.0 & 0.0 & 0.0 \\
Cefazolin & 61.5 & 0.0 & 38.5 \\
Cefepime & 61.5 & 15.4 & 23.1 \\
Gentamicin & 61.5 & 0.0 & 38.5 \\
Ciprofloxacin & 23.0 & 0.0 & 77.0 \\
Aztreonam & 53.8 & 0.0 & 46.2 \\
Levofloxacin & 38.4 & 7.6 & 54.0 \\
Imipenem & 69.2 & 0.0 & 30.7 \\
Cotrimoxazole & 61.5 & 0.0 & 38.5 \\
Ampicillin/sulbactam & 38.4 & 15.4 & 46.2 \\
Piperacillin/tazobactam & 69.2 & 0.0 & 31.8 \\
Ceftriaxone & 37.5 & 0.0 & 62.5 \\
Tobramycin & 53.8 & 0.0 & 46.2 \\
\hline
\end{tabular}

$\mathrm{mL}$, the sensitivity, specificity, positive likelihood ratio, and negative likelihood ratio for sepsis prognosis were $52.11 \%$, $76.23 \%, 2.19$, and 0.63 , respectively (Table 9).

\section{Discussion}

Sepsis is a life-threatening organ dysfunction caused by an infection-induced dysregulated inflammatory response. ${ }^{12}$ Symptoms and signs in early-stage sepsis are insidious and non-specific; further, biomarkers for clinical auxiliary examinations have unsatisfactory sensitivity and specificity, which impedes early recognition and intervention. Consequently, sepsis rapidly progresses, which causes higher mortality and disability rates in the advanced stages. In developed countries, the incidence of sepsis ranges from $66 / 100,000$ to $300 / 1$ million while the mortality is $27 \%-$ $36 \%{ }^{13,14}$ In China, sepsis accounts for $37.3 \%$ of ICU admissions and is the leading cause of ICU deaths, with more than 1 million annual deaths. ${ }^{15}$ This retrospective study analyzed the clinical data, laboratory indicators, influencing factors, 
Table 8 Multivariate Logistic Regression Analysis Results of Sepsis in Patients with Sepsis

\begin{tabular}{|l|l|l|l|l|}
\hline Independent Variable & B & OR & $\mathbf{9 5 \%}$ Cl & P \\
\hline Consciousness & 1.027 & 2.792 & $0.725-10.745$ & 0.135 \\
SOFA & 0.140 & 1.150 & $0.992-1.333$ & 0.064 \\
PT & -0.003 & 0.997 & $0.966-1.029$ & 0.846 \\
INR & 0.251 & 1.285 & $0.693-2.385$ & 0.426 \\
FIB & -0.103 & 0.902 & $0.789-1.030$ & 0.128 \\
D-D & 0.091 & 1.096 & $1.030-1.166$ & 0.004 \\
AST & 0.000 & 1.000 & $0.999-1.001$ & 0.954 \\
\hline
\end{tabular}

and common pathogens in 329 patients with sepsis; moreover, we conducted drug susceptibility tests. Our results could guide the clinical diagnosis and treatment of sepsis.

The SOFA score allows assessment of the severity of organ dysfunction/failure in patients with sepsis, which can dynamically reflect changes in the condition. Acharya et al reported that the SOFA score can sufficiently reflect the sepsis severity, which could allow the evaluation of organ dysfunction in patients with sepsis. ${ }^{16}$ Toker et al reported that the SOFA score has strong sensitivity and predictive utility in the sepsis diagnosis. ${ }^{17}$ The SOFA score has a high discriminant ability, which allows the prediction of emergency and Hospital mortality. We found that the SOFA score can significantly predict the poor prognosis of sepsis, ie, a higher SOFA score indicated a worse prognosis $(P<0.05)$.

Microbial culture is crucially involved in the diagnosis and treatment of sepsis. Timely and accurate microbial culturing allows the determination of the source and pathogen of infection, which facilitates targeted medication and drug selection after empirical treatment; moreover, it is closely associated with treatment success. However, relevant studies have mainly focused on bacterial sepsis, with $30-60 \%$ of patients with sepsis have negative blood cultures; moreover, some studies ${ }^{18}$ have attributed this to viral infections. Phua et al reported that the proportion of negative cultures in patients with sepsis was as high as $42 \%$, which could be due to viral infections. ${ }^{19}$ We isolated 47 pathogenic strains from blood culture samples, including 29 gram-positive (61.7\%), 18 gram-negative (38.3\%),

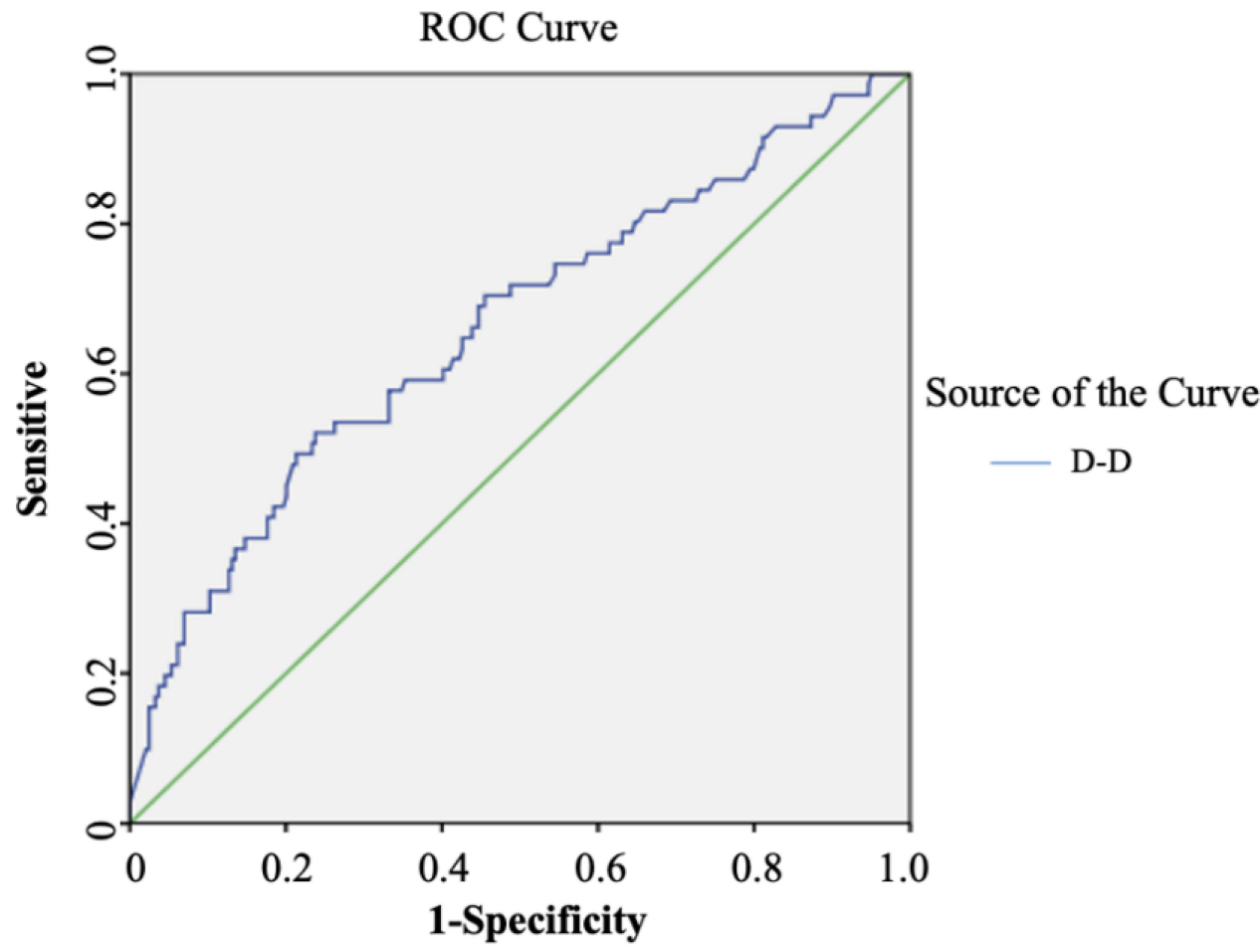

Figure I ROC curve of D-dimer levels for predicting sepsis outcome. 
Table 9 ROC Curve Analysis Results for D-Dimer Levels

\begin{tabular}{|l|l|}
\hline Indicators & D-D \\
\hline AUC $(95 \% \mathrm{Cl})$ & $0.66 \mathrm{I}(0.586-0.736)$ \\
The critical value & $3.36 \mu \mathrm{g} / \mathrm{mL}$ \\
The sensitivity & $52.11 \%$ \\
Specific degrees & $76.23 \%$ \\
Positive likelihood ratio & 2.19 \\
Negative likelihood ratio & 0.63 \\
\hline
\end{tabular}

respectively. The most common gram-negative pathogens in blood culture were Klebsiella pneumoniae and Escherichia coli, while the most common gram-positive pathogens were Staphylococcus aureus and Staphylococcus hominis. Gram positive cocci showed 100\% resistance to penicillin and low resistance to rifampicin, vancomycin and linezolid. Gram-negative bacteria were $100 \%$ susceptible to amikacin and $77 \%$ and $62.5 \%$ resistant to ciprofloxacin and ceftriaxone respectively. Overuse of antibiotics, as well as misuse and inappropriate antibiotic prescribing, are the main causes of multidrug resistance (MDR), especially in developing countries. With the widespread use of antibiotics and the increasing availability of antimicrobial drugs, pathogenic bacteria have become significantly more resistant to antimicrobial drugs even in a multi-drug resistant manner. ${ }^{20}$ The misuse of antibiotics also greatly increases the likelihood and duration of drug-bacteria contact, with sensitive bacteria being gradually replaced by resistant bacteria during prolonged drug-bacteria contact. Antibiotic management in hospitals can be effective in reducing antimicrobial resistance. In addition, there is a need to revise clinical guidelines, which should include steps to justify the continuation of antibiotic therapy and criteria for selecting antibiotics at the start of treatment.

The positive rate of blood culture was not high because patients with sepsis who had negative blood cultures had been treated with antibiotics 48 hours prior to diagnosis. ${ }^{21}$ Patients with sepsis with negative and positive blood cultures showed similar characteristics. Those with negative blood cultures had been exposed to antibiotics 48 hours prior to sample collection, including 47 cases (14.3\%) with meropenem, 35 cases (10.6\%) with cephalosporin antibiotics and 124 cases $(37.7 \%)$ with unknown drugs. Therefore, there is a need for further large-scale studies.

Sepsis occurrence and development are often associated with disorders of the fibrinolytic coagulation system; moreover, severe sepsis is closely associated with the occurrence of hemostatic defects and disseminated intravascular coagulation. ${ }^{22} \mathrm{D}$-dimer is a degraded product during fibrinolysis. Specifically, D-dimer is formed through fibrinolysininduced degradation of cross-linked fibrous proteins, with D-dimer levels reflecting the fibrinolysis state of the body. Fabrizio et al proposed that D-dimer generation is dependent on both coagulation and fibrinolysis, as well as other variables alone or in combination, which makes it a prognostic factor for patients with sepsis. ${ }^{23}$ Zhan et al reported that D-dimer levels could independently predict 28-day mortality in patients with sepsis, which allowed risk stratification. ${ }^{24}$ In this study, D-dimer levels could predict the outcome of patients with sepsis, where higher D-dimer levels indicate worse outcomes of patients with sepsis.

\section{Conclusion}

In summary, the main pathogenic bacteria detected in adult patients with sepsis in Wenzhou, china were Klebsiella pneumoniae, Escherichia coli, Staphylococcus aureus, and Staphylococcus hominis. The rational use of antibiotics based on the principles of antimicrobial medication and drug susceptibility tests can prevent the abuse of antibiotics and allow good therapeutic effects. D-dimer levels could independently predict the prognosis of sepsis in adults.

\section{Abbreviations}

SOFA, Sequential Organ Failure Assessment; ICU, intensive care units; CRP, C-reactive protein; PCT, procalcitonin; ROC, receiver operating characteristic. 


\section{Data Sharing Statement}

The datasets generated and analyzed during the current study are available from the corresponding author on reasonable request.

\section{Ethics Approval and Consent to Participate}

The study was approved by the Ethical Committee of the Second Affiliated Hospital of Wenzhou Medical University (Approval No: LCKY2020-421). Written informed consent were obtained from the patients, and this study was conducted in accordance with the Declaration of Helsinki.

\section{Acknowledgments}

The results published here are completely based on the data detected by the Department of Clinical Laboratory. We thank them for their contributions.

\section{Author Contributions}

All authors made a significant contribution to the work reported, whether that is in the conception, study design, execution, acquisition of data, analysis and interpretation, or in all these areas; took part in drafting, revising or critically reviewing the article; gave final approval of the version to be published; have agreed on the journal to which the article has been submitted; and agreed to be accountable for all aspects of the work.

\section{Funding}

This work was partially supported by grants from the Natural Science Foundation of China (NSFC81601849), Zhejiang Provincial Medicine and Health Technology Project (2019RC217), the Natural Science Foundation of Zhejiang Provincial (Y22H162326).

\section{Disclosure}

The authors report no conflicts of interest in this work.

\section{References}

1. Singer M, Deutschman CS, Seymour CW, et al. The third international consensus definitions for sepsis and septic shock (Sepsis-3). JAMA. 2016;315(8):801-810. doi:10.1001/jama.2016.0287

2. van der Poll T, van de Veerdonk FL, Scicluna BP, et al. The immunopathology of sepsis and potential therapeutic targets. Nat Rev Immunol. 2017;17(7):407-420. doi:10.1038/nri.2017.36

3. Mayr FB, Yende S, Angus DC. Epidemiology of severe sepsis. Virulence. 2014;5(1):4-11. doi:10.4161/viru.27372

4. Rudd KE, Johnson SC, Agesa KM, et al. Global, regional, and national sepsis incidence and mortality, 1990-2017: analysis for the Global Burden of Disease Study. Lancet. 2020;395(10219):200-211. doi:10.1016/S0140-6736(19)32989-7

5. Pierrakos C, Vincent J-L. Sepsis biomarkers: a review. Crit Care. 2010;14(1):1-18. doi:10.1186/cc8872

6. Maurer FP, Christner M, Hentschke M, et al. Advances in rapid identification and susceptibility testing of bacteria in the clinical microbiology laboratory: implications for patient care and antimicrobial stewardship programs. Infect Dis Rep. 2017;9(1):6839. doi:10.4081/idr.2017.6839

7. Cao Y, Chai YF, Deng Y. Guidelines for emergency treatment of sepsis/septic shock in China (2018). Infect Inflamm Repair. 2019;20(01):3-22.

8. Vincent J-L, de Mendonca A, Cantraine F, et al. Use of the SOFA score to assess the incidence of organ dysfunction/failure in intensive care units: results of a multicenter, prospective study. Crit Care Med. 1998;26(11):1793-1800. doi:10.1097/00003246-199811000-00016

9. Rhodes A, Evans LE, Alhazzani W, et al. Surviving sepsis campaign: international guidelines for management of sepsis and septic shock: 2016. Intensive Care Med. 2017;43(3):304-377. doi:10.1007/s00134-017-4683-6

10. Anonymous. Technical guidelines for clinical trials of antimicrobial drugs. Chin J Clin Pharmacol. 2014;9:844-856.

11. Patel JB, Cockerill F, Bradford PA. Performance standards for antimicrobial susceptibility testing: twenty-fifth informational supplement. Clin Lab Stand Inst. 2015;35:29-50.

12. Shankar-Hari M, Phillips GS, Levy ML, et al. Developing a new definition and assessing new clinical criteria for septic shock: for the Third International Consensus Definitions for Sepsis and Septic Shock (Sepsis-3). JAMA. 2016;315(8):775-787. doi:10.1001/jama.2016.0289

13. Stoll BJ, Hansen NI, Bell EF, et al. Trends in care practices, morbidity, and mortality of extremely preterm neonates, 1993-2012. JAMA. 2015;314 (10):1039-1051. doi:10.1001/jama.2015.10244

14. Keeley A, Hine P, Nsutebu E. The recognition and management of sepsis and septic shock: a guide for non-intensivists. Postgrad Med J. 2017;93 (1104):626-634. doi:10.1136/postgradmedj-2016-134519

15. Cecconi M, Evans L, Levy M, et al. Sepsis and septic shock. Lancet. 2018;392(10141):75-87. doi:10.1016/S0140-6736(18)30696-2 
16. Acharya S, Pradhan B, Marhatta M. Application of" the Sequential Organ Failure Assessment (SOFA) score" in predicting outcome in ICU patients with SIRS. Kathmandu Univ Med J. 2007;5(4):475-483.

17. Toker AK, Kose S, Turken M. Comparison of SOFA score, SIRS, qSOFA, and qSOFA+ L criteria in the diagnosis and prognosis of sepsis. Eurasian J Med. 2021;53(1):40. doi:10.5152/eurasianjmed.2021.20081

18. Research SAIDC. Causes and outcomes of sepsis in Southeast Asia: a multinational multicentre cross-sectional study. Lancet Glob Health. 2017;5 (2):e157-e167.

19. Phua J, Ngerng WJ, See KC, et al. Characteristics and outcomes of culture-negative versus culture-positive severe sepsis. Crit Care. 2013;17 (5):1-12. doi:10.1186/cc12896

20. López Romo A, Quirós R. Appropriate use of antibiotics: an unmet need. Ther Adv Urol. 2019;11:1756287219832174. doi:10.1177/ 1756287219832174

21. Sigakis MJ, Jewell E, Maile MD, et al. Culture negative and culture positive sepsis: a comparison of characteristics and outcomes. Anesth Analg. 2019;129(5):1300. doi:10.1213/ANE.0000000000004072

22. Bonaventura A, Carbone F, Vecchié A, et al. The role of resistin and myeloperoxidase in severe sepsis and septic shock: results from the ALBIOS trial. Eur J Clin Invest. 2020;50(10):e13333. doi:10.1111/eci.13333

23. Semeraro F, Ammollo CT, Caironi P, et al. D-dimer corrected for thrombin and plasmin generation is a strong predictor of mortality in patients with sepsis. Blood Transfus. 2020;18(4):304. doi:10.2450/2019.0175-19

24. Zhan ZG, Li CS. [Prognostic value of D-dimer in patients with sepsis in emergency department: a prospective study]. Chin Crit Care Med. 2012;24 (3):135-139. Chinese.

\section{Publish your work in this journal}

Infection and Drug Resistance is an international, peer-reviewed open-access journal that focuses on the optimal treatment of infection (bacterial, fungal and viral) and the development and institution of preventive strategies to minimize the development and spread of resistance. The journal is specifically concerned with the epidemiology of antibiotic resistance and the mechanisms of resistance development and diffusion in both hospitals and the community. The manuscript management system is completely online and includes a very quick and fair peer-review system, which is all easy to use. Visit http://www.dovepress.com/testimonials.php to read real quotes from published authors. 